\title{
Togitare
}

\section{BACTÉRIAS RESISTENTES ISOLADAS DE SUPERFÍCIES INANIMADAS EM UM HOSPITAL PÚBLICO}

\author{
Emanuelle Righetto Corrêa ${ }^{1}$ 우 \\ Alexandre Paulo Machado ${ }^{1}$ (i) \\ Juliano Bortolini ${ }^{1}$ (i) \\ Jocilene de Carvalho Miraveti ${ }^{1}$ (1) \\ Liliana Victorino Alves Corrêa ${ }^{1}$ (i) \\ Marília Duarte Valim
}

\section{RESUMO}

Objetivo: descrever o perfil de resistência a antimicrobianos de microrganismos presentes em superfícies inanimadas.

Método: estudo descritivo, realizado entre fevereiro e junho de 2018. Coletou-se 40 amostras microbiológicas de superfícies da Clínica Médica e Unidade de Terapia Intensiva Adulto em um hospital de Mato Grosso, Brasil. A identificação e sensibilidade microbiana foram realizadas através do VITEK-2. A análise dos resultados de resistência foi avaliada conforme as diretrizes do Clinical Laboratory Standards Institute.

Resultados: foram isolados 32 microrganismos das 22 amostras contaminadas, dentre eles 14 (43,8\%) Staphylococcus coagulase negativa, sete $(21,9 \%)$ Acinetobacter baumanni complex, três $(9,4 \%)$ Enterobacter aerogenes. Dentre os Staphylococcus coagulase negativa, $11(78,6 \%)$ apresentaram multirresistência a antimicrobianos e três $(42,9 \%)$ dos isolados de Acinetobacter baumanni complex foram extremamente resistentes.

Conclusão: evidenciou-se a necessidade de educação com ênfase na desinfecção correta e frequente de superfícies e na higienização das mãos após tocar o paciente e as superfícies próximas a ele.

DESCRITORES: Infecção Hospitalar; Segurança do Paciente; Contaminação de Equipamentos; Resistência Microbiana a Medicamentos; Serviço Hospitalar de Limpeza.

\section{BACTERIAS RESISTENTES AISLADAS DE SUPERFICIES INANIMADAS EN UN HOSPITAL PÚBLICO}

\section{RESUMEN:}

Objetivo: describir el perfil de resistencia de microrganismos presentes en superficies inanimadas a agentes antimicrobianos. Método: estudio descriptivo realizado entre febrero y junio de 2018. Se recolectaron 40 muestras microbiológicas de superficies de la Clínica Médica y de la Unidad de Cuidados Intensivos para Adultos de un hospital de Mato Grosso, Brasil. Los procesos de identificación y sensibilidad microbiana se realizaron a través del dispositivo VITEK 2. El análisis de los resultados de resistencia se evaluó conforme a las directrices del Clinical Laboratory Standards Institute. Resultados: se aislaron 32 microrganismos de las 22 muestras contaminadas; entre ellos, hubo 14 (43,8\%) Staphylococcus coagulasa negativa, siete (21,9\%) Acinetobacter baumanni complex y tres $(9,4 \%)$ Enterobacter aerogenes. Entre los Staphylococcus coagulasa negativa, 11 (78,6\%) presentaron multi-resistencia a agentes antimicrobianos y tres (42,9\%) de los aislados bacterianos de Acinetobacter baumanni complex fueron extremamente resistentes. Conclusión: se hizo evidente la necesidad de instrucción con énfasis en la correcta y frecuente desinfección de superficies y en el lavado de manos después de entrar en contacto con el paciente y con las superficies próximas al paciente.

DESCRIPTORES: Infección Hospitalaria; Seguridad del Paciente; Contaminación de Equipos; Resistencia Microbiana a Medicamentos; Servicio Hospitalario de Limpieza. 
As Infecções Relacionadas à Assistência à Saúde (IRAS) estão entre os eventos adversos mais frequentes em pacientes hospitalizados, e resultam em altos índices de morbidade e mortalidade em todo o mundo, com consequente aumento do tempo de internação e dos custos hospitalares(1). Estudo europeu multicêntrico observou que, dos 151.709 pacientes internados em unidades de terapia intensiva (UTI) por mais de dois dias, $8,4 \%$ apresentaram pelo menos um quadro de IRAS ${ }^{(2)}$. No Brasil, a relevância da problemática dessas infecções pode ser constatada em investigação que observou em 377 pacientes diagnosticados com IRAS uma taxa de mortalidade de $20,7 \%$, de janeiro de 2015 a julho de $2016^{(3)}$.

A contaminação do ambiente desempenha um papel importante na aquisição de patógenos nosocomiais, tanto por pacientes quanto por profissionais da saúde. Os profissionais frequentemente adquirem microrganismos patogênicos devido ao contato direto com os pacientes, com fluidos corporais ou com superfícies ambientais contaminadas $^{(4)}$. Os agentes patogênicos podem sobreviver em superfícies ambientais por dias, semanas e até meses quando não são realizadas a limpeza e a desinfecção correta, aumentando substancialmente o risco de IRAS ${ }^{(5)}$.

Estudos confirmam que bactérias multirresistentes têm sido relatadas como microrganismos contaminantes de superfícies, telefones, teclados, equipamentos hospitalares comumente usados e superfícies frequentemente tocadas em UT(1,6-9). Os profissionais de saúde geralmente subestimam o papel das superfícies ambientais na transmissão de IRAS. Nesse sentido, estudos demonstraram que os profissionais comumente não realizam a higiene das mãos (HM) após o contato em superfícies inanimadas no entorno de um paciente, embora seja frequente a indicação para HM nesse momento na prática assistencial(10).

Ressalta-se que, principalmente em países em desenvolvimento, há poucos dados sobre a extensão da contaminação e perfil microbiano de equipamentos utilizados com frequência e superfícies inanimadas em unidades de saúde $e^{(11)}$. Diante disso, o objetivo deste estudo foi descrever o perfil de resistência a antimicrobianos de microrganismos presentes em superfícies inanimadas do ambiente e de equipamentos hospitalares de uma Unidade de Terapia Intensiva Adulto e Clínica Médica de um hospital.

\section{MÉTODO}

Trata-se de estudo transversal, descritivo, realizado no período de fevereiro a junho de 2018, em uma unidade de Clínica Médica e em uma UTI adulto de um hospital público de Cuiabá-MT. Esses setores foram escolhidos por serem locais críticos para a disseminação de IRAS, visto que apresentam grande quantidade de procedimentos invasivos e utilização frequente de antibióticos, além de abrigarem pacientes com imunidade comprometida.

Para avaliação microbiológica, foram aleatoriamente selecionadas 40 amostras (20 da Clínica Médica e 20 da UTI) de superfícies ambientais e de equipamentos hospitalares. Foram incluídos no presente estudo superfícies inanimadas e equipamentos hospitalares que pertencessem aos respectivos setores e que apresentassem alta frequência de contato, seja por profissionais, pacientes ou acompanhantes. Foram selecionadas amostras de teclados, maçanetas, bomba de infusão, monitores multiparâmetros, mesa de cabeceira, grade lateral do leito, chão, torneira, glicosímetro, capa de prontuário, tampa de lixeira, frasco de dieta, frasco de aspiração, dispensador de sabonete, mouse de computador e 
apoio de braço de cadeira. Ademais, foram incluídas amostras dos pisos situados próximos ao leito do paciente.

Os procedimentos para coleta foram realizados sem informar aos profissionais de qual superfície do ambiente seria coletada a amostra, a fim de reproduzir o cenário real da prática. Como instrumento de coleta de dados, foi utilizado um questionário no qual - pesquisador respondeu se a superfície ou equipamento pertenciam ao setor e se estes apresentavam alta frequência de contato com profissionais, pacientes ou acompanhantes. Além disso, nesse instrumento foram identificados a data, o setor da coleta e a superfície inanimada ou do equipamento que foram realizados a coleta da amostra.

Para coleta das amostras, foram utilizados swabs esterilizados com meio de transporte Stuart, que foram friccionados nas superfícies dos objetos e seus relevos, devidamente codificados, e encaminhados para processamento e análise em laboratório de microbiologia. Os swabs foram então semeados por inoculação direta em placas de petri contendo meios seletivos para o crescimento de microrganismos: ágar seletivo para Enterococcus, ágar Cetrimida, ágar Azul de Metileno Eosina, ágar Mueller- Hinton suplementado com 4\% de $\mathrm{NaCl}$ e seis $\mu \mathrm{g} / \mathrm{mL}$ de oxacilina, ágar Mueller-Hinton suplementado com $70 \mu \mathrm{g} / \mathrm{mL}$ de sulfato de zinco e um $\mu \mathrm{g} / \mathrm{mL}$ de meropenem, e ágar batata dextrose com gentamicina $40 \mathrm{mg} / \mathrm{L}$. As placas foram incubadas a $36^{\circ} \mathrm{C}$ e a leitura ocorreu em 24,48 e 72 horas. As colônias foram identificadas primeiramente pelas características morfotintoriais e por técnicas microbiológicas clássicas.

Aidentificação e o teste de suscetibilidade antimicrobiana de isolados foram realizados de forma automatizada por meio do equipamento VITEK $2 \circledR$ (bioMerieux $®$, Marcy L'Etoile, França) de acordo com as instruções do fabricante. A concentração inibitória mínima (CIM) para análise dos resultados de resistência foi avaliada conforme as diretrizes do Clinical Laboratory Standards Institute de $2018^{(12)}$.

Os dados coletados foram duplamente digitados em uma planilha do Excel para evitar erros de transcrição. Para análise estatística descritiva e inferencial, utilizou-se o software $\mathrm{R}^{(13)}$. Realizou-se o teste $\mathrm{Z}$ para análise comparativa de proporções de crescimento de microrganismos entre os setores estudados. O nível de significância considerado na análise foi de $5 \%$.

O estudo foi submetido ao Comitê de Ética em Pesquisa do Hospital Universitário Julio Muller e aprovado com parecer número 2.441.333.

\section{RESULTADOS}

Dentre as 40 amostras coletadas, 22 (55\%) apresentaram crescimento positivo para pelo menos um microrganismo. A descrição das superfícies ambientais e de equipamentos que foram amostrados, bem como a quantidade de amostras positivas para crescimento bacteriano e a identificação e número de bactérias isoladas, são apresentados na Tabela 1. 
Tabela 1 - Tipo e número de superfícies inanimadas amostradas $(n=40)$ para microrganismos. Cuiabá, MT, Brasil, 2018

\section{Superfícies inanimadas}

\section{Amostras positivas Microrganismos isolados (quantidade para crescimento de amostras positivas) bacteriano}

\begin{tabular}{|c|c|c|}
\hline Piso $(n=5)$ & 4 & $\begin{array}{l}\text { CoNS (2), Acinetobacter baumanni } \\
\text { complex (3), Enterobacter aerogenes (1) } \\
\text { e Enterococcus hirae (1) }\end{array}$ \\
\hline Mesa de cabeceira $(n=1)$ & 1 & $\begin{array}{l}\text { CoNS (1), Acinetobacter baumanni } \\
\text { complex (1) e Enterococcus faecium (1) }\end{array}$ \\
\hline Tampa de lixeira com defeito $(n=2)$ & 1 & $\begin{array}{l}\text { CoNS (1), Acinetobacter baumanni (1) e } \\
\text { Enterobacter aerogenes (1) }\end{array}$ \\
\hline Monitor multiparâmetro $(n=2)$ & 1 & Acinetobacter baumanni complex (1) \\
\hline Dispensador de sabonete $(n=1)$ & 1 & Acinetobacter baumanni complex (1) \\
\hline Grade de leito $(n=4)$ & 2 & $\begin{array}{l}\text { CoNS (2), Enterobacter asburiae (1) e } \\
\text { Enterobacter aerogenes (1) }\end{array}$ \\
\hline $\begin{array}{l}\text { Torneira da sala de preparo de } \\
\text { medicação }(n=2)\end{array}$ & 2 & $\begin{array}{l}\text { CoNS (1) e Sphingomonas paucimobilis } \\
\text { (1) e Roseomonas gilardii (1) }\end{array}$ \\
\hline Maçaneta $(n=4)$ & 2 & CoNS (2) e Burkholderia spp (1) \\
\hline Teclado $(n=3)$ & 2 & $\begin{array}{l}\text { CoNS (1) e Sphingomonas paucimobilis } \\
\text { (1) }\end{array}$ \\
\hline Telefone $(n=2)$ & 1 & Staphylococcus aureus (1) \\
\hline Apoio de braço de cadeira $(n=1)$ & 1 & Staphylococcus aureus (1) \\
\hline Bomba de infusão $(n=5)$ & 3 & CoNS (3) \\
\hline Glicosímetro portátil $(n=1)$ & 1 & CoNS (1) \\
\hline Bancada de preparo de medicação $(n=2)$ & - & - \\
\hline Mouse de computador $(n=2)$ & - & - \\
\hline Capa de prontuário $(n=1)$ & - & - \\
\hline Frasco de aspiração $(n=1)$ & - & - \\
\hline
\end{tabular}

Frasco de dieta enteral $(n=1)$

CoNS - Staphylococcus Coagulase Negativa

Nota: Mais de um microrganismo esteve presente em algumas amostras.

Fonte: Autores (2018).

Destas amostras foram isoladas e identificadas 32 espécies bacterianas, consistindo em $14(43,8 \%)$ amostras positivas para Staphylococcus coagulase negativa (CoNS), sete $(21,9 \%)$ para Acinetobacter baumanni complex, três $(9,4 \%)$ para Enterobacter aerogenes, duas $(6,3 \%)$ para Staphylococcus aureus. Além disso, identificou-se nas superfícies inanimadas ambientais e de equipamentos hospitalares amostrados um isolado de Enterococcus hirae, Enterococcus faecium, Enterobacter asburie, Sphingomonas paucimobilis, Roseomonas gilardii e Burkholderia spp. É importante salientar que o método de identificação automatizada VITEK $2 \otimes$ identifica apenas como Acinetobacter baumanni complex, sendo assim qualquer uma dessas espécies podem ser patogênicas, tais como $A$. baumannii, $A$. calcoaceticus, A. nosocomialis, A. dijkshoorniae e A. pittii(14).

Dentre as amostras coletadas com isolados CoNS, cinco $(35,7 \%)$ apresentaram 
Staphylococcus haemolyticus, três (21,4\%) Staphylococcus homnis spp., duas (14,3\%) Staphylococcus epidermidis e em duas (14,3\%) amostras observou-se Staphylococcus saprophyticus. Ademais, isolados de Staphylococcus lentus e Staphylococcus captis foram encontrados em uma $(7,1 \%)$ amostra cada um.

Com relação à suscetibilidade antimicrobiana, todos os isolados de CoNS apresentaram sensibilidade à vancomicina, linezolida, daptomicina, teicoplanina, tigecilina, nitrofurantoína e estreptomicina. No entanto, cabe destacar, conforme Tabela 2, que todos os isolados de CoNS foram resistentes à benzilpenicilina e, do total dos 14 isolados, apenas três foram suscetíveis à oxacilina e à clindamicina, correspondendo a uma resistência de $78,6 \%$ dos CoNS a cada um desses antibióticos. Dentre os dois isolados de S. aureus recuperados das amostras coletadas, um foi resistente à benzilpenicilina, oxacilina, eritromicina, gentamicina, levofloxacino e clindamicina, enquanto o outro isolado de $S$. aureus foi resistente apenas à benzilpenicilina (Tabela 2).

Tabela 2 - Perfil de resistência de bactérias Gram-positivas ( $n=18)$ encontradas em amostras de superfícies inanimadas. Cuiabá, MT, Brasil, 2018

\begin{tabular}{lcccccccc} 
Variáveis & \multicolumn{2}{c}{$\begin{array}{c}\text { CoNS } \\
(\mathbf{n}=\mathbf{1 4})\end{array}$} & $\begin{array}{c}\text { Staphylococcus } \\
\text { aureus }(\mathbf{n = 2})\end{array}$ & $\begin{array}{c}\text { Enterococcus } \\
\text { faecium }(\mathbf{n = 1})\end{array}$ & $\begin{array}{c}\text { Enterococcus } \\
\text { hirae }(\mathbf{n}=\mathbf{1})\end{array}$ \\
\cline { 2 - 12 } & $\mathbf{f}$ & $\%$ & $\mathbf{f}$ & $\%$ & $\mathbf{f}$ & $\%$ & $\mathbf{f}$ & $\%$ \\
\hline Benzilpenicilina & 14 & 100 & 2 & 100 & 1 & 100 & 0 & 0 \\
\hline Oxacilina & 11 & 78,6 & 1 & 50 & 0 & 0 & 0 & 0 \\
\hline Gentamicina & 6 & 42,9 & 1 & 50 & 0 & 0 & 0 & 0 \\
\hline Levofloxacina & 10 & 71,4 & 1 & 50 & 1 & 100 & 0 & 0 \\
\hline Eritromicina & 9 & 64,3 & 1 & 50 & 1 & 100 & 0 & 0 \\
\hline Clindamicina & 11 & 78,6 & 1 & 50 & 1 & 100 & 1 & 100 \\
\hline Rifampicina & 4 & 28,6 & 0 & 50 & 0 & 0 & 0 & 0 \\
\hline Trimetroprim/Sulfametoxazol & 6 & 42,9 & 1 & 50 & 1 & 100 & 0 & 0 \\
\hline Ampicilina & - & - & - & - & 1 & 100 & 0 & 0 \\
\hline Estreptomicina & - & - & - & - & 1 & 100 & 0 & 0
\end{tabular}

- teste de sensibilidade não realizado.

Fonte: Autores (2018).

A Tabela 3 mostra o perfil de resistência do bacilos gram-negativos recuperados das amostras pesquisadas. Ressalta-se que, dentre os sete isolados de Acinetobacter baumanni complex, três apresentaram resistência a carbapenêmicos (meropenem e imipenem), no entanto foram sensíveis à colistina. Não foi realizado teste de suscetibilidade dos isolados Sphingomonas paucimobilis, Roseomonas gilardii e Burkholderia. 
Tabela 3 - Perfil de resistência de bactérias Gram-negativas $(n=11)$ encontradas em amostras de superfícies inanimadas. Cuiabá, MT, Brasil, 2018

\begin{tabular}{lcccccc} 
Variáveis & $\begin{array}{c}\text { Acinetobacter } \\
\text { baumanni complex } \\
(\mathbf{n = 7})\end{array}$ & $\begin{array}{c}\text { Enterobacter } \\
\text { aerogenes } \\
(\mathbf{n = 3})\end{array}$ & $\begin{array}{c}\text { Enterobacter } \\
\text { asburiae } \\
(\mathbf{n = 1})\end{array}$ \\
\cline { 2 - 7 } & $\mathbf{f}$ & $\%$ & $\mathbf{f}$ & $\%$ & $\mathbf{f}$ & $\%$ \\
\hline Ampicilina & 7 & 100 & 3 & 100 & 0 & 0 \\
\hline Cefuroxima & 7 & 100 & 3 & 100 & 0 & 0 \\
\hline Cefuroxima axetil & 7 & 100 & 3 & 100 & 0 & 0 \\
\hline Cefoxitina & 7 & 100 & 3 & 100 & 1 & 100 \\
\hline Ceftazidima & 3 & 42,9 & 0 & 0 & 0 & 0 \\
\hline Ceftriaxona & 3 & 42,9 & 0 & 0 & 0 & 0 \\
\hline Ampicilina/Sulbactam & 2 & 28,6 & 3 & 100 & 0 & 0 \\
\hline Piperaciclina/Tazobactam & 4 & 57,1 & 0 & 0 & 0 & 0 \\
\hline Cefepima & 3 & 42,9 & 0 & 0 & 0 & 0 \\
\hline Imipenem & 3 & 42,9 & 0 & 0 & 0 & 0 \\
\hline Meropenem & 3 & 42,9 & 0 & 0 & 0 & 0 \\
\hline Amicacina & 3 & 42,9 & 0 & 0 & 0 & 0 \\
\hline Gentamicina & 1 & 14,3 & 0 & 0 & 0 & 0 \\
\hline Ciprofloxacina & 3 & 42,9 & 0 & 0 & 0 & 0 \\
\hline Tigeciclina & 1 & 14,3 & 0 & 0 & 0 & 0 \\
\hline Colistina & 0 & 0 & 0 & 0 & 0 & 0
\end{tabular}

Fonte: Autores (2018).

O Quadro 1 apresenta o perfil das bactérias isoladas das amostras do estudo multirresistentes (MDR) e extremamente resistentes (XDR) a antimicrobianos. MDR é definida como resistência adquirida a pelo menos um agente em três ou mais categorias antimicrobianas. Já a XDR é conceituada como a resistência a pelo menos um agente em todas categorias antimicrobianas, exceto apenas uma ou duas categorias ${ }^{(15)}$.

Quadro 1 - Perfil de resistência a antimicrobianos de microrganismos MDR ou XDR. Cuiabá, MT, Brasil, 2018 (continua)

\begin{tabular}{|c|c|c|}
\hline Isolado bacteriano & $\begin{array}{c}\text { Número de } \\
\text { microrganismos } \\
\text { MDR ou XDR }\end{array}$ & Padrão de resistência \\
\hline $\begin{array}{c}\text { Acinetobacter } \\
\text { baumanni complex }\end{array}$ & $\begin{array}{c}\text { XDR - 3/7 } \\
(42,9 \%)\end{array}$ & $\begin{array}{c}\text { AMP, APS (beta-lactâmicos), CEF, CFT (cefalosporinas de } \\
2^{\text {a }} \text { geração), CFZ, CFX (cefalosporinas de 3 }{ }^{\text {a }} \text { geração), PIT } \\
\text { (beta-lactâmico inibidores de } \beta \text {-lactamase) CPM- cefepima } \\
\text { (cefalosporina 4 } \\
\text { GEN geração), IMP, MPM (carbapenêmicos), AMI, } \\
\text { GENinoglicosídeos), CIP (quinolona) }\end{array}$ \\
\hline
\end{tabular}




\begin{tabular}{|c|c|c|}
\hline $\begin{array}{c}\text { Staphylococcus } \\
\text { coagulase negativa }\end{array}$ & $\begin{array}{c}\text { MDR - 10/14 } \\
(71,4 \%)\end{array}$ & $\begin{array}{c}\text { BEN (beta-lactâmico), OXA (beta-lactâmico penicilinase- } \\
\text { resistente), CLI (lincosamida), LVX (fluorquinolona), ERI } \\
\text { (macrolídeo) }\end{array}$ \\
\hline $\begin{array}{c}\text { Staphylococcus } \\
\text { aureus }\end{array}$ & $\begin{array}{c}\text { MDR }-1 / 2 \\
(50 \%)\end{array}$ & $\begin{array}{c}\text { BEN (beta-lactâmico), OXA (beta-lactâmico penicilinase- } \\
\text { resistente), CLI (lincosamida), LVX (fluorquinolona), ERI } \\
\text { (macrolídeo), GEN (aminoglicosídeo) }\end{array}$ \\
\hline $\begin{array}{c}\text { Enterococcus } \\
\text { faecium }\end{array}$ & $\begin{array}{c}\text { MDR - 1/1 } \\
(100 \%)\end{array}$ & $\begin{array}{c}\text { ERI (macrolídeos), LVX (fluorquinolona), CLI (lincosamida), SUT } \\
\text { (sulfonamida+ diamino-pirimidina, EST (aminoglicosídeo) }\end{array}$ \\
\hline
\end{tabular}

MDR- multirresistente, XDR- Extremamente resistente, BEM- Benzilpenicilina, OXA- Oxacilina, CLI- Clindamicina, LVX- levofloxacina, ERI- eritromicina, AMP- Ampicilina, APS- Ampicilina/Sulbactam, CEF- Cefuroxima, CFT- Cefoxitina, CFZ- Ceftazidima, CFXCeftriaxona, CPM- Cefepima,, PIT- Piperaciclina/Tazobactam, GEN- Gentamicina, CIP- Ciprofloxacina, SUT- Sulfametoxazoltrimetropim, EST- Estreptromicina, AMI- Amicacina, MPM- Meropenem, IPM- Imipenem.

Fonte: Autores (2018).

Cabe destacar que $10(71,4 \%)$ isolados de CoNS apresentaram perfil fenotípico de MDR e que três amostras apresentaram isolados de $A$. baumanni complexXDR, apresentando suscetibilidade a apenas tigeciclina e colistina. $O$ três isolados de $A$. baumanni complex XDR foram recuperados em ambiente próximo ao paciente em amostras da mesa de cabeceira, do piso e do monitor multiparâmetro. $O$ isolado MDR de E. faecium foi encontrado em amostra da mesa de cabeceira do paciente. E a amostra coletada de um telefone do posto de enfermagem da Clínica Médica foi positiva para S. aureus MDR. Os isolados de E. aerogenes, E. asburie e Enterococcus hirae não apresentaram perfil fenotípico de MDR.

Conforme a Tabela 4, a Clínica Médica foi o setor que apresentou mais amostras contaminadas, apresentando $65 \%$ das suas amostras com crescimento de microrganismos, no entanto, essa diferença entre setores não foi estatisticamente significante $(p=0,34)$. Ressalta-se que na Clínica Médica estão seis amostras positivas para Acinetobacter baumanii complex, sendo três com isolados XDR.

Tabela 4 - Distribuição dos microrganismos presentes no ambiente segundo setor do hospital. Cuiabá, MT, Brasil, 2018

\begin{tabular}{|c|c|c|c|c|c|c|c|c|c|c|c|c|c|c|c|c|}
\hline \multirow[t]{2}{*}{ Variáveis } & \multicolumn{2}{|c|}{$\begin{array}{c}\text { BGN } \\
(n=14)\end{array}$} & \multicolumn{2}{|c|}{$\begin{array}{l}\text { CoNS } \\
(n=14)\end{array}$} & \multicolumn{2}{|c|}{$\begin{array}{c}\text { MRSA } \\
(n=1)\end{array}$} & \multicolumn{2}{|c|}{$\begin{array}{c}\text { MSSA } \\
(n=1)\end{array}$} & \multicolumn{2}{|c|}{$\begin{array}{c}\text { E. } \\
\text { faecium } \\
(n=1)\end{array}$} & \multicolumn{2}{|c|}{$\begin{array}{c}\text { E. hirae } \\
(n=1)\end{array}$} & \multicolumn{2}{|c|}{$\begin{array}{c}\text { Com } \\
\text { crescimento } \\
(n=22)\end{array}$} & \multicolumn{2}{|c|}{$\begin{array}{c}\text { Sem } \\
\text { crescimento } \\
(n=18)\end{array}$} \\
\hline & $f$ & $\%$ & $f$ & $\%$ & $f$ & $\%$ & $f$ & $\%$ & $f$ & $\%$ & $f$ & $\%$ & $f$ & $\%$ & $f$ & $\%$ \\
\hline $\begin{array}{l}\text { Clínica Médica } \\
(n=20)\end{array}$ & 10 & 50 & 9 & 45 & 1 & 5 & - & - & 1 & 5 & 1 & 5 & 13 & 65 & 7 & 35 \\
\hline $\begin{array}{l}\text { UTI Adulto } \\
(n=20)\end{array}$ & 4 & 20 & 5 & 25 & - & - & 1 & 5 & - & - & - & - & 9 & 45 & 11 & 55 \\
\hline
\end{tabular}


A mesa de cabeceira foi a amostra com maior variedade de microrganismos em uma única amostra, inclusive com isolados bacterianos de $E$. faecium e CoNS MDR e A. baumanni XDR. Essa alta contaminação da mesa de cabeceira foi encontrada em investigações anteriores ${ }^{(4,13)}$. Estudo realizado na Etiópia encontrou, em 27 amostras de mesas de cabeceiras, 13 isolados de microrganismos, sendo um CoNS, três Escherichia coli, duas Klebsiella, dois Proteus, duas Pseudomonas aeruginosa, duas Serratia e um $S$. aureus ${ }^{(11)}$. No Irã, pesquisa com amostra maior de mesas de cabeceiras (124) verificou que havia $22,58 \%$ de $S$. epidermidis, $10,48 \%$ de A. baumanni, 5,65\% de S. aureus ${ }^{(4)}$.

A grade lateral da cama, monitor multiparâmetro e bomba de infusão também apresentaram microrganismos frequentemente relacionados às IRAS e relevantes para o aumento das taxas de morbimortalidade, tais como A. baumanniresistente a carbapenêmicos, Enterobacter spp, CoNS resistente à oxacilina. Em estudos similares também se verificaram patógenos no ambiente do paciente $(4,6,11,16)$. Estudo anterior verificou em 128 amostras de camas $14(10,9 \%)$ S. aureus, 25 (19,5\%) CoNS, 16 (12,5\%) Acinetobacter spp ${ }^{(4)}$.

Nesse contexto, é preocupante a quantidade de bactérias patogênicas em superfícies ambientais e equipamentos hospitalares nos arredores dos pacientes, uma vez que estes são frequentemente tocados e mutuamente contatados por profissionais, pacientes e visitantes, favorecendo desse modo a transmissão cruzada(17). Pesquisa observacional verificou que os dois itens mais tocados mutuamente por profissionais, pacientes e visitantes foram as grades laterais dos leitos, com média de 13,6 contato-episódios por hora, seguidas por mesas de cabeceira com 12,3 contato-episódios por hora(17).

Os equipamentos que servem de apoio para o processo de trabalho das equipes assistenciais e administrativas, tais como telefone, apoio de braço da cadeira, teclado de computador, dispensador de sabonete na sala de preparo de medicação, apresentaram amostras positivas para bactérias com potencial patogênico, inclusive S. aureus MDR. Esse fato indica que os profissionais podem ser veículos de potenciais patógenos de pacientes para ambientes mais distantes da assistência.

A contaminação das superfícies inanimadas situadas fora do ambiente do paciente corrobora com os resultados de pesquisas realizadas em hospitais no Brasil e Irã ${ }^{(4,18)}$. Essas superfícies e equipamentos, mesmo não sendo utilizados na assistência direta ao paciente, podem ser capazes de colonizar e infectar o paciente através das mãos dos profissionais e, por constituírem equipamentos de uso coletivo, representam um elevado potencial para dispersão da contaminação microbiana ${ }^{(18)}$.

No que se refere às coletas de amostras de tampas de lixeiras com o acionamento de levantamento da tampa com defeitos, foram observados isolados de $A$. baumanni complex e Staphylococcus saprophyticus. Sendo necessário levantá-la manualmente após a HM, consequentemente, o profissional poderia ser contaminado por essas bactérias e disseminá-las pelo hospital. Vale ressaltar que iniciativas que obtiveram diminuição dos índices de IRAS investiram primeiramente em infraestrutura ${ }^{(19)}$.

As amostras do piso apresentaram diversidade de bactérias, corroborando com pesquisas anteriores ${ }^{(11,20)}$. Mesmo o piso apresentando altos índices de contaminação, as equipes de limpeza comumente demonstram dar pouca relevância à sua desinfecção. No entanto, os pisos são um reservatório potencial de transmissão, visto que, frequentemente, entram em contato com objetos que são posteriormente tocados pelas mãos dos pacientes ou dos profissionais ${ }^{(20)}$.

Nesse sentido, pesquisa realizada em cinco hospitais com 318 amostras de pisos, em quartos de pacientes, identificou contaminação com Clostridium difficile, MRSA e Enterococcus resistente à vancomicina (VRE). Nessa mesma investigação, constatou-se que houve transferência de patógenos do chão para objetos que estavam em contato com o chão e, destes, para as mãos e luvas, e foram encontrados $18 \%$ de MRSA, $6 \%$ de VRE e $3 \%$ de C. difficile ${ }^{(20)}$. 
Quanto aos isolados de CoNS encontrados nas amostras, constatou-se índice de resistência alto de $71,4 \%$ à oxacilina. Destaca-se a grande quantidade de amostras com isolados de CoNS com fenótipo MDR, uma vez que essa característica confere patogenicidade a essas bactérias, o que se torna um desafio para o tratamento de infecções adquiridas em ambientes hospitalares ${ }^{(21)}$.

A contaminação no ambiente hospitalar por isolados de CoNS MDR é preocupante também, pelo fato de que esses microrganismos podem ser importantes reservatórios de genes de resistência a antimicrobianos, os quais podem ser transferidos entre espécies estafilocócicas ${ }^{(22)}$. Cabe salientar que foi observada multirresistência de um isolado de S. aureus resistente à oxacilina, o qual, por sua vez, causa bacteremias que estão relacionadas a uma maior mortalidade e tempo prolongado de internação(23).

Com relação à suscetibilidade dos $B G N$, vale assinalar a gravidade de três amostras apresentarem isolados de $A$. baumanni complex XDR, visto que as infecções por estes microrganismos causam morbimortalidade significativa entre pacientes hospitalizados e ainda não se tem um tratamento ideal estabelecido ${ }^{(23)}$. Essa elevada resistência encontrada nesses isolados pode estar relacionado ao uso generalizado de carbapenêmicos ${ }^{(24)}$.

No que concerne à presença de amostras com isolados bacterianos nos diferentes setores do hospital, pesquisa anterior verificou maior índice de contaminação de CoNS resistente à oxacilina na Clínica Médica do que na UTI, o que corrobora com os achados do presente estudo(25). Nesse sentido, pode-se inferir que, na Clínica Médica, comumente há maior contingente de pacientes, familiares e subdimensionamento de profissionais de enfermagem e de limpeza, o que pode explicar os resultados encontrados. Estudo recente brasileiro verificou como potenciais fatores para limpeza ambiental insatisfatória, a baixa eficiência do biocida usado, panos contaminados, conformidade variável ao procedimento de $\mathrm{HM}$ e estabilidade de vários gêneros de bactérias à desinfecção(26).

Quanto à limitação desta pesquisa, está o número reduzido de amostras e de setores de coletas, o que impede generalizações dos resultados.

\section{CONCLUSÃO}

Espera-se que esta pesquisa possa ser utilizada pelas equipes de Prevenção e Controle de Infecções Relacionadas à Assistência à Saúde, para o planejamento de programas de educação que enfatizem a importância da limpeza e desinfecção corretas, frequentes e rotineiras de equipamentos e superfícies inanimadas das unidades de saúde. Ademais, a presença de bactérias resistentes em superfícies situadas em ambiente mais distante do paciente reforça a importância da higiene das mãos após tocar o paciente e após contato com as superfícies inanimadas situadas nos seus arredores, atitude esta que também deve ser incentivada insistentemente pelas equipes de educação continuada.

\section{AGRADECIMENTOS}

Agradecemos à Fundação de Amparo à Pesquisa do Estado de Mato Grosso (FAPEMAT), processo número 0214392/2017, pelo financiamento desta pesquisa. 
1. Agencia Nacional de Vigilância Sanitária (ANVISA). Gerência de Vigilância e Monitoramento em Serviços de Saúde. Gerência Geral de Tecnologia em Serviços de Saúde. Medidas de Prevenção de Infecção Relacionada à Assistência à Saúde. Série Segurança do Paciente e Qualidade em Serviços de Saúde. 2. ed. [Internet]. Brasília (DF): Ministério da Saúde; 2017 [acesso em 12 ago 2017]. Disponível em: http://www.riocomsaude.rj.gov.br/Publico/MostrarArquivo.aspx?C=pCiWUy84\%2BR0\%3D.

2. European Centre for Disease Prevention and Control (ECDC). Healthcare-associated infections acquired in intensive care units. In: ECDC. Annual epidemiological report for 2016. Stockholm: ECDC [Internet]. 2018 [acesso em 21 jul 2017]. Disponível em: https://www.ecdc.europa.eu/sites/default/files/documents/ AER for 2016-HAI 0.pdf.

3. Soares SG de SC, Mascarenhas MDM, Moura LNB de, Machado AFP. Caracterização das infecções relacionadas à assistência à saúde em um hospital de ensino do Nordeste do Brasil. Rev Enferm UFPI [Internet]. 2017 [acesso em 06 jun 2018]; 6(2). Disponível em: https://doi.org/10.26694/reufpi.v6i2.5933.

4. Tajeddin E, Rashidan M, Razaghi M, Javadi SSS, Sherafat SJ, Alebouyeh M, et al. The role of the intensive care unit environment and health-care workers in the transmission of bacteria associated with hospital acquired infections. J Infect Public Health [Internet]. 2016 [acesso em 12 nov 2017]; 9(1). Disponível em: https://doi.org/10.1016/j.jiph.2015.05.010.

5. Kramer A, Schwebke I, Kampf G. How long do nosocomial pathogens persist on inanimate surfaces? A systematic review. BMC Infect Dis [Internet]. 2006 [acesso em 16 ago 2017]; 6(130). Disponível em: https://doi.org/10.1186/1471-2334-6-130.

6. Rocha IV, Ferraz P de M, Farias TGS de, Oliveira SR de. Resistência de bactérias isoladas em equipamentos em unidade de terapia intensiva. Acta paul. enferm [Internet]. 2015 [acesso em 04 dez 2019]; 28(5). Disponível em: https://doi.org/10.1590/1982-0194201500073.

7. Costa DM, Johani K, Melo DS, Lopes LKO, Lima LKOL, Tipple AFV, et al. Biofilm contamination of high-touched surfaces in intensive care units: epidemiology and potential impacts. Lett Appl Microbiol [Internet]. 2019 [acesso em 05 dez 2019]; 68(4). Disponível em: https://doi.org/10.1111/lam.13127.

8. Adams CE, Smith J, Watson V, Robertson C, Dancer SJ. Examining the association between surface bioburden and frequently touched sites in intensive care. J Hosp Infect [Internet]. 2017 [acesso em 21 jan 2018]; 95(1). Disponível em: https://doi.org/10.1016/j.jhin.2016.11.002.

9. Price JR, Cole K, Bexley A, Kostiou V, Eyre DW, Golubchik T, et al. Transmission of Staphylococcus aureus between health-care workers, the environment, and patients in an intensive care unit: a longitudinal cohort study based on whole-genome sequencing. Lancet Infect Dis [Internet]. 2017 [acesso em 04 fev 2018]; 17(2). Disponível em: https://doi.org/10.1016/S1473-3099(16)30413-3.

10. Krishna A, Navalkele B, Pervaiz A, Kotecha A, Maroof S, Stern D, et al. Monitoring hand hygiene compliance among healthcare workers at a tertiary care center: use of secret observers is the way forward. Open Forum Infect Dis [Internet]. 2017 [acesso em 02 fev 2018]; 4(suppl.1). Disponível em: https://doi.org/10.1093/ofid/ofx163.1023.

11. Worku T, Derseh D, Kumalo A. Bacterial Profile and Antimicrobial Susceptibility Pattern of the Isolates from Stethoscope, Thermometer, and Inanimate Surfaces of Mizan-Tepi University Teaching Hospital, Southwest Ethiopia. Int J Microbiol [Internet]. 2018 [acesso em 18 fev 2019]; 2018:9824251. Disponível em: https://doi.org/10.1155/2018/9824251.

12. Clinical Laboratory Standards Institute. Performance standards for antimicrobial susceptibility testing. 28. ed. Wayne (PA): CLSI; 2018.

13. $R$ Core Team. R: a language and environment for statistical computing [Internet]. Vienna: R Foundation for Statistical Computing; 2018 [acesso em 13 fev 2019]. Disponível em: https://www.R-project.org/.

14. Wang J, Ruan Z, Feng Y, Fu Y, Jiang Y, Wang H, et al. Species distribution of clinical Acinetobacter isolates revealed by different identification techniques. PLoS One [Internet]. 2014 [acesso em 21 fev 2019]; 9(8). Disponível em: https://doi.org/10.1371/journal.pone.0104882. 
15. Magiorakos AP, Srinivasan A, Carey RB, Carmeli Y, Falagas ME, Giske CG, et al. Multidrug-resistant, extensively drug-resistant and pandrug-resistant bacteria: an international expert proposal for interim standard definitions for acquired resistance. Clin Microbiol Infect [Internet]. 2012 [acesso em 07 jun 2019]; 18(3). Disponível em: https://doi.org/10.1111/j.1469-0691.2011.03570.x.

16. Visalachy S, Palraj KK, Kopula SS, Sekar U. Carriage of multidrug resistant bacteria on frequently contacted surfaces and hands of health care workers. J Clin Diagn Res [Internet]. 2016 [acesso em 10 fev 2018]; 10(5). Disponível em: https://doi.org/10.7860/JCDR/2016/19692.7772.

17. Cheng VCC, Chau PH, Lee WM, Ho SKY, Lee DWY, So SYC, et al. Hand-touch contact assessment of high-touch and mutual-touch surfaces among healthcare workers, patients, and visitors. J Hosp Infect [Internet]. 2015 [acesso em 11 fev 2018]; 90(3). Disponível em: https://doi.org/10.1016/j.jhin.2014.12.024.

18. Cordeiro ALAO, Oliveira MMC, Fernandes JD, Barros CSMA, Castro LMC. Contaminação de equipamentos em unidade de terapia intensiva. Acta paul enferm. [Internet]. 2015 [acesso em 22 out 2019]; 28(2). Disponível em: https://doi.org/10.1590/1982-0194201500027.

19. Fariñas-Alvarez C, Portal-María T, Flor-Morales V, Aja-Herrero A, Fabo-Navarro M, Lanza-Marín S, et al. Estrategia multimodal para la mejora de la adherencia a la higiene de manos en un hospital universitario. Rev Calid Asist [Internet]. 2017 [acesso em 12 fev 2018]; 32(1). Disponível em: https://doi.org/10.1016/j. cali.2016.06.011.

20. Deshpande A, Cadnum JL, Fertelli D, Sitzlar B, Thota P, Mana TS, et al. Are hospital floors an underappreciated reservoir for transmission of health care-associated pathogens? Am J Infect Control [Internet]. 2017 [acesso em 27 jan 2018]; 45(3). Disponível em: https://doi.org/10.1016/j.ajic.2016.11.005.

21. Du X, Zhu Y, Song Y, Li T, Luo T, Sun G, et al. Molecular analysis of Staphylococcus epidermidis strains isolated from community and hospital environments in China. PloS one [Internet]. 2013 [acesso em 27 jan 2018]; 8(5). Disponível em: https://doi.org/10.1371/journal.pone.0062742.

22 Becker K, Heilmann C, Peters G. Coagulase-negative staphylococci. Clin. microbiol. rev. [Internet]. 2014 [acesso em 15 jun 2019]; 27(4). Disponível em: https://doi.org/10.1128/cmr.00109-13.

23. Smidt MCF de, Singh-Moodley A, Badat R, Quan V, Kularatne R, Nana T, et al. Staphylococcus aureus bacteraemia in Gauteng academic hospitals, South Africa. Int J Infect Dis [Internet]. 2015 [acesso em 13 set 2019]; 30. Disponível em: https://doi.org/10.1016/j.jijid.2014.10.011.

24. Batirel A, Balkan II, Karabay O, Agalar C, Akalin S, Alici O, et al. Comparison of colistin-carbapenem, colistin-sulbactam, and colistin plus other antibacterial agents for the treatment of extremely drugresistant Acinetobacter baumannii bloodstream infections. Eur J Clin Microbiol Infect Dis [Internet]. 2014 [acesso em 27 jul 2019]; 33. Disponível em: https://doi.org/10.1007/s10096-014-2070-6.

25. Seng R, Kitti T, Thummeepak R, Kongthai P, Leungtongkam U, Wannalerdsakun S, et al. Biofilm formation of methicillin-resistant coagulase negative staphylococci (MR-CoNS) isolated from community and hospital environments. PloS one [Internet]. 2017 [acesso em 26 jun 2019]; 12(8). Disponível em: https://doi.org/10.1371/journal.pone.0184172.

26. Ribeiro LF, Lopes EM, Kishi LT, Ribeiro LFC, Menegueti MG, Gaspar GG, et al. Microbial community profiling in intensive care units expose limitations in current sanitary standards. Front Public Health [Internet]. 2019 [acesso em 02 out 2019]; 7(240). Disponível em: https://doi.org/10.3389/ fpubh.2019.00240. 
COMO REFERENCIAR ESTE ARTIGO:

Corrêa ER, Machado AP, Bortolini J, Miraveti J de C, Corrêa LVA, Valim MD. Bactérias resistentes isoladas de superfícies inanimadas em um hospital público. Cogitare enferm. [Internet]. 2021 [acesso em "colocar data de acesso, dia, mês abreviado e ano"]; 26. Disponível em: http://dx.doi.org/10.5380/ce.v26i0.74774.

*Artigo extraído da dissertação de mestrado "Adesão à higiene das mãos e a ocorrência de microrganismos potencialmente patogênicos". Universidade Federal de Mato Grosso, 2019.

Recebido em: 27/06/2020

Aprovado em: 11/12/2020

Editora associada: Luciana Alcântara Nogueira

Autor Correspondente:

Emanuelle Righetto Corrêa

Universidade Federal de Mato Grosso - Cuiabá, MT, Brasil

E-mail: emanuellerighetto@gmail.com

Contribuição dos autores:

Contribuições substanciais para a concepção ou desenho do estudo; ou a aquisição, análise ou interpretação de dados do estudo - ERC, APM, JB, JCM, LVAC, MDV

Elaboração e revisão crítica do conteúdo intelectual do estudo - ERC, APM, MDV

Aprovação da versão final do estudo a ser publicado - ERC, APM, JB, JCM, LVAC, MDV

Responsável por todos os aspectos do estudo, assegurando as questões de precisão ou integridade de qualquer parte do estudo - ERC, MDV

Copyright $\odot 2021$ Este é um artigo em acesso aberto distribuído nos termos da Licença Creative Commons Atribuição, que permite o uso irrestrito, a distribuição e reprodução em qualquer meio desde que o artigo original seja devidamente citado. 